\title{
Follow-up Analysis to Geminga's Contribution to the Local Positron Excess with the HAWC gamma-ray Observatory
}

\author{
Ramiro Torres-Escobedo, ${ }^{a, *}$ Hao Zhou, ${ }^{a}$ Eduardo de la Fuente, ${ }^{b, c}$ for the HAWC \\ Collaboration and Mattia Di Mauro ${ }^{d}$ \\ ${ }^{a}$ Tsung-Dao Lee Institute \& School of Physics and Astronomy, Shanghai Jiao Tong University \\ Shanghai, China \\ ${ }^{b}$ Departamento de Física, CUCEI, Universidad de Guadalajara \\ Jalisco, México \\ ${ }^{c}$ Institute for Cosmic Ray Research, University of Tokyo, Kashiwa Campus \\ Chiba, Japan \\ ${ }^{d}$ Istituto Nazionale di Fisica Nucleare (INFN) \\ Torino, Italy \\ E-mail: torresramiro350@sjtu.edu.cn, hao_zhou@sjtu.edu.cn, \\ eduardo.delafuente@academicos.udg.mx, dimauro.mattia@gmail.com
}

Two cosmic-ray experiments, PAMELA and AMS-02, measured an abnormal positron excess above $10 \mathrm{GeV}$. This excess is well understood, but it has been considered direct evidence of dark matter. However, this excess could be produced by nearby pulsars too. The HAWC collaboration previously studied the extended gamma-ray emission of two nearby pulsars, Geminga and PSR B0656+14, but found no significant contribution to this excess from these pulsars. The previous study of HAWC led to the reinterpretation of our result and initiated the concept of inverse Compton (IC) halos. Fitting a new halo model and 1343 days of data from the HAWC gamma-ray observatory may better constrain the contribution of these pulsars to the positron excess. This halo model utilizes 3D templates of gamma-ray emission from electron IC interactions to fit the diffusion coefficient and electron injection spectral index. This model can further help to study the energy-dependent diffusion and incorporate anisotropic diffusion with the proper motion of the pulsar.

$37^{\text {th }}$ International Cosmic Ray Conference (ICRC 2021)

July 12th-23rd, 2021

Online - Berlin, Germany

\footnotetext{
${ }^{*}$ Presenter
} 


\section{Introduction}

Pulsar wind nebulae are considered natural sites of efficient electron and positron emission with particle acceleration powered by the pulsar or surrounding nebulae. Their $e^{-} e^{+}$emission is estimated to contribute largely to the leptonic Galactic cosmic ray flux. This contribution could help explain observations of an excess in the positron fraction above an energy of $10 \mathrm{GeV}$ measured by PAMELA [1], Fermi-LAT [2], AMS-02 [3]. The nature of the mechanism responsible for this anomalous excess is not well understood. However, work from several people suggests the origin of this excess is a consequence of dark matter particle mechanisms: annihilation/decay or nearby astrophysical sources-pulsar wind nebulae[4][5].

The HAWC collaboration reported the first observations of two extended gamma-ray regions coinciding with the sky locations of two middle-aged pulsars, Geminga and PSR B0656+14 (Monogem) [6]. These two sources were candidates for the study of their contribution to the local positron excess. The extended gamma-ray emission was associated with inverse Compton (IC) interactions as $e^{-} e^{+}$escaped into the interstellar medium via diffusion. The HAWC collaboration presented a spectral and spatial study of Geminga and Monogem with 500 days of data. The results demonstrated high efficiency in conversion of pulsar spin-down energy to $e^{-} e^{+}$emission but derived a diffusion coefficient that was 100 times smaller than the average galactic value. This result suggests that if such a slow diffusion region extends to Earth, then any $e^{-} e^{+}$emission from Geminga and Monogem is insufficient to explain this abnormal positron excess [7].

This study introduced a new class of gamma-ray sources referred to as " $\mathrm{TeV}$ halos". TeV halos differ from pulsar wind nebulae as they can extend up to a few tens of parsecs [8]. The extended gamma-ray regions result from $e^{-} e^{+}$IC interactions with low energy fields as they escape into the interstellar medium (ISM) via diffusion. Work from [9] at GeV energies with Fermi-LAT observed the halos around Geminga and Monogem and confirmed the presence of a slow diffusion region up to 100 parsecs. The recent observations from [10] measured region slow diffusion at $\sim 160 \mathrm{TeV}$ region for halo around PSR J0622 +3749 which claims slow diffusion is a property of TeV halos.

This contribution discusses a new halo model for studying two TeV halos, Geminga and Monogem, with the HAWC observatory. The new halo model permits the study of the diffusion coefficient, injection spectral index, and efficiency of pulsar spin-down energy to $e^{-} e^{+}$emission. This model was used on a 1343 day HAWC data set, which shows an improvement over the result from HAWC.

\section{Model}

This model utilizes three-dimensional templates of diffuse gamma-ray emission from inverse Compton (IC) interactions with spectral and spatial information for a pulsar. Each pixel in this template contains values with units of flux $1 /\left(\mathrm{MeV} \mathrm{cm}^{2} \mathrm{~s} s \mathrm{~s}\right)$ for different energy bins in the range of $100 \mathrm{GeV}-100 \mathrm{TeV}$. The templates use flux estimates from [9] for different injection electron/positron spectral indices and diffusion coefficients (see Figure 1). The efficiency in conversion of pulsar spin-down energy to $e^{-} e^{+}, K\left(\dot{E} \rightarrow e^{-} e^{+}\right)$is assumed to be $100 \%$.

We investigated the effect of diffusion coefficient on the spectra of the gamma-ray emission, which required one-dimensional spectral templates with fluxes integrated for different regions 

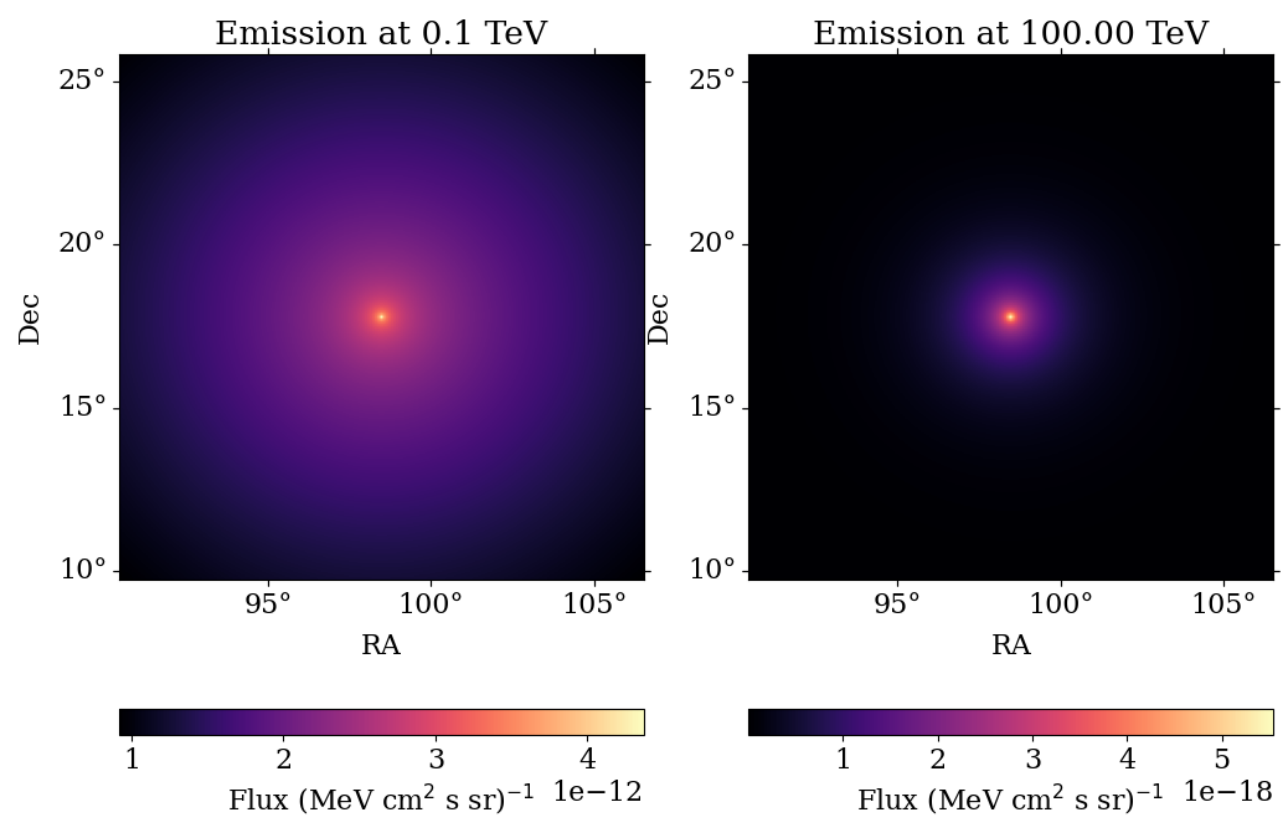

Figure 1: Templates of gamma-ray emission with a diffusion coefficient $D_{0}=1.58 \mathrm{~cm}^{2} / \mathrm{s}$ at $1 \mathrm{GeV}$ for a injection spectral index of 2.0. The region of gamma-ray emission at $0.1 \mathrm{TeV}$ shows a larger extent than at $100 \mathrm{TeV}$ because of the significant $e^{-} e^{+}$energy losses.

around the pulsar. This effect showed that high diffusion coefficients require large regions for full containment of gamma-ray emission, which reflected flux values differing by almost an order of magnitude. The impact on the gamma-ray spectra became negligible for a region of 20-30 degrees. With this study, it was possible to separate the relationship between diffusion coefficient and injection spectral index. Furthermore, the flux values for a region of the 30-degree region were used to normalize the $3 \mathrm{D}$ template pixel values, leaving them with $1 /(\mathrm{sr})$ units.

We propose a new halo model that builds a table of 3D normalized templates, which permits the interpolation over different diffusion coefficients and injection spectral indices over normalized flux values. The interpolated values are further interpolated over the template parameters: energy, RA, and Dec. The fitting parameters for the analysis are then diffusion coefficient, injection spectral index, and $K\left(\dot{E} \rightarrow e^{-} e^{+}\right)$. The above model provides a custom spatial shape to study the morphology of $\mathrm{TeV}$ halo candidates with a custom spectral shape from the aforementioned spectral templates. 


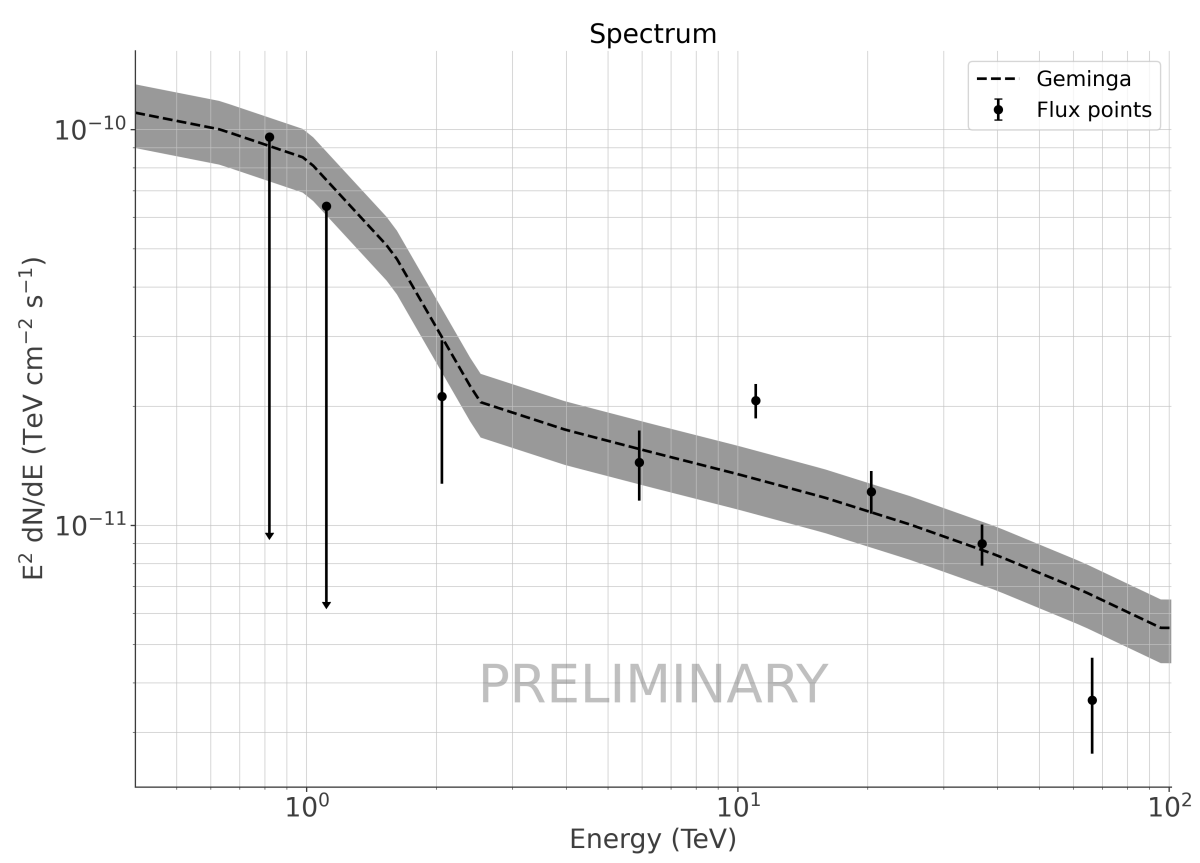

Figure 2: Geminga spectrum obtained with implemented halo model. First flux points show rescaled flux values from 30-degree region to a 8-degree region. Change in slope $\sim 2 \mathrm{TeV}$ is a consequence of continuous injection that becomes important above this energy. The fitting parameters for Geminga are diffusion coefficient, spectral index, and $K\left(\dot{E} \rightarrow e^{-} e^{+}\right)$. The gray band shows the statistical errors from the analysis.

\section{High Altitude Water Cherenkov Observatory}

The HAWC gamma-ray observatory is on Sierra Negra Volcano at an elevation of 4,100 meters in Puebla State, Mexico. The HAWC site houses 300 water Cherenkov detectors (WCDs), each with dimensions 7.3 meters in diameter and 5 meters in height. A WCD holds up to 220,000 liters of ultra-purified water with four photomultipliers at its base that detect Cherenkov light emitted by charged particles from air showers. HAWC has an effective area of detection of 22,000 $\mathrm{m}^{2}$, which grants a field of view of $2 \pi$ and can observe $2 / 3$ of the northern sky with a $>95 \%$ duty cycle.

The wide field of view of HAWC makes it the ideal instrument to study extended sources like Geminga and Monogem. The first derived result for diffusion coefficient was obtained with 500 days of data after its full operational deployment in March 2015, with data binned by the fraction of the detector hit during the event. We report the first preliminary results from a newly implemented $\mathrm{TeV}$ halo model on the halos around of Geminga and Monogem with 1343 days of HAWC data that use the ground parameter technique [11].

\section{Results \& Discussion}

The analysis used the halo model with templates for diffusion coefficients of $10^{25}-10^{28} \mathrm{~cm}^{2} / \mathrm{s}$ at $1 \mathrm{GeV}$ and spectral indices of $1.5-2.4$. This range allowed for thorough sampling of the fitting parameters. The resulting spectrum for Geminga is shown in Figure 2. 


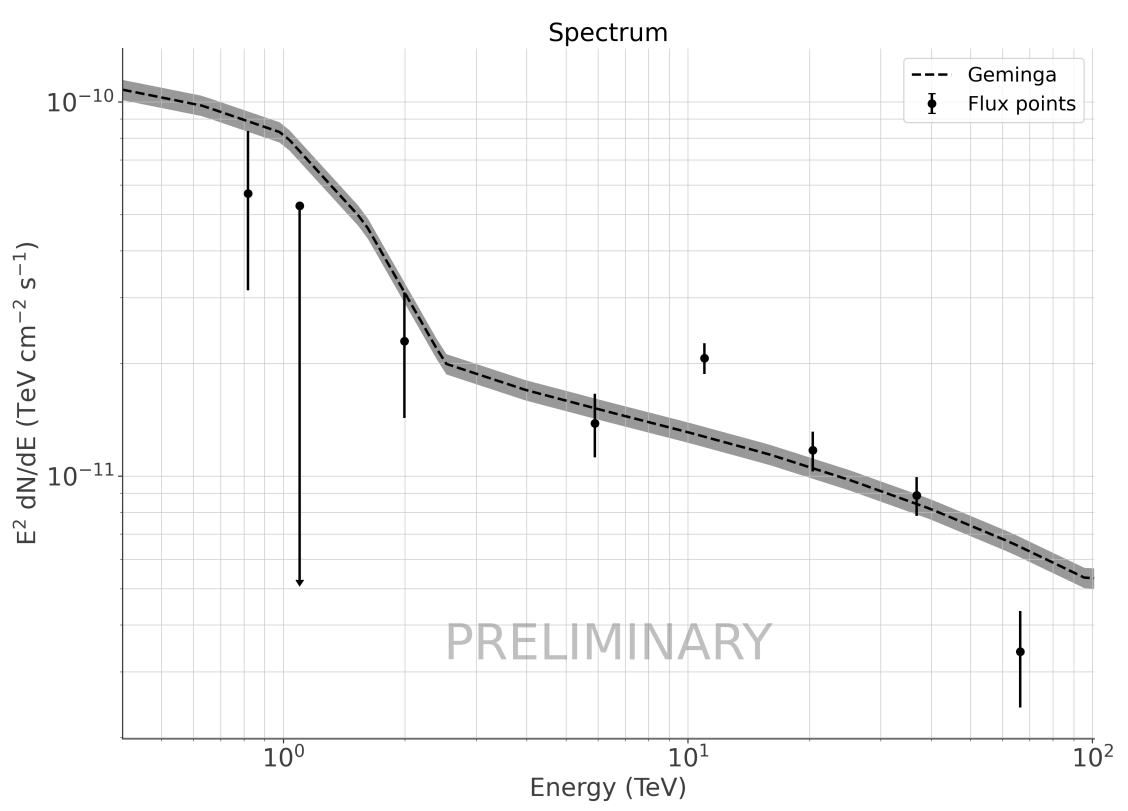

(a) Geminga

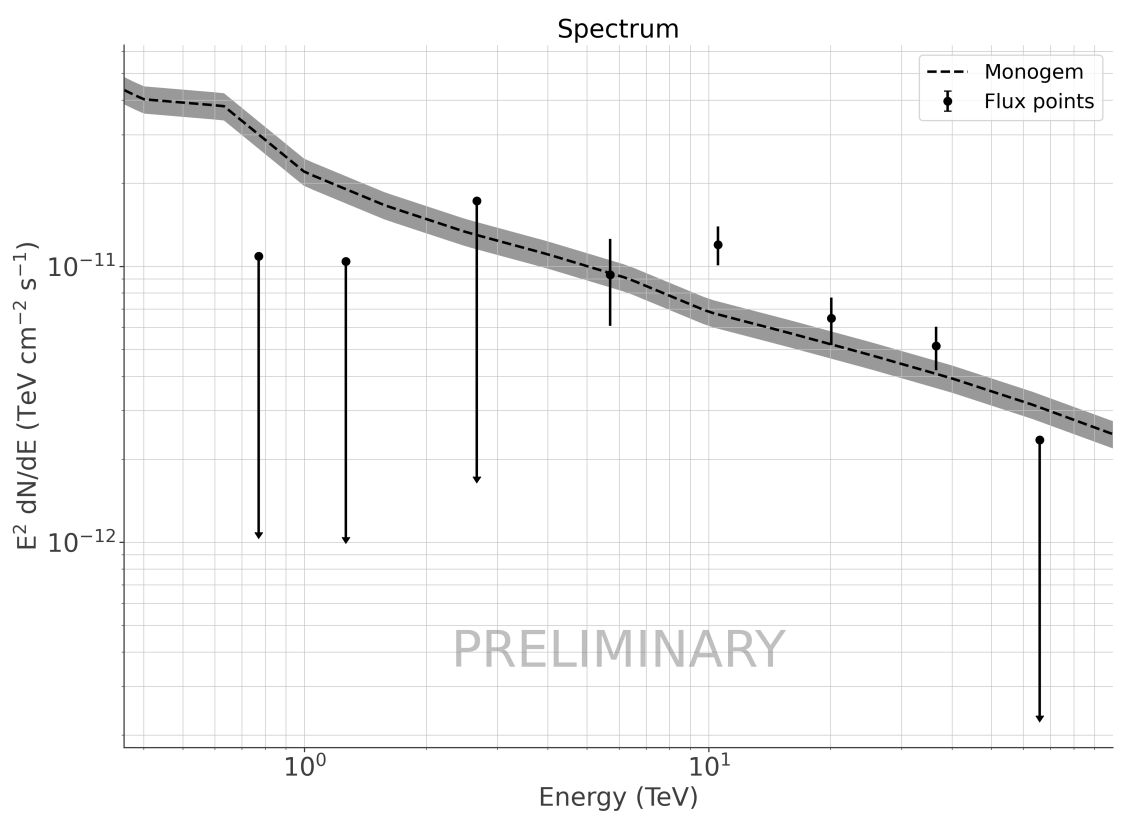

(b) Monogem

Figure 3: Joint fit of Geminga and Monogem using the halo interpolation model. The diffusion coefficient and spectral index for both sources were fixed to the best-fit results from the Geminga only analysis. The only fitting parameters are the $K\left(\dot{E} \rightarrow e^{-} e^{+}\right)$for Geminga and Monogem.

The first feature to notice is the upper limits for energies $\sim 1 \mathrm{TeV}$. These flux values are not "true" detections by HAWC, but as mentioned in section 2, large diffusion coefficients require large regions to contain diffuse gamma-ray emission fully. Therefore, these flux values are rescaled from a 30-degree region to that of 8 degrees. Secondly, a change in slope at $\sim 2 \mathrm{TeV}$ is a consequence 
of continuous diffuse injection that becomes important above the maximum energy of the source under a burst-like assumption. This value can be calculated by

$$
\varepsilon_{\text {source, } \max }=\frac{1}{b \cdot t_{\text {age }}},
$$

where $\mathrm{b}$ represents the $e^{-} e^{+}$sources and $t_{\text {age }}$ is the age of the source. This change in slope is typical of positron spectra, but it shows at comparable energies in gamma-ray spectra because of the large energy losses that $e^{-} e^{+}$as they diffuse through the ISM.

The best-fitting results are presented at the conference but show good agreement with the derived diffusion coefficient by the HAWC collaboration. The diffusion coefficient and spectral index values obtained from Geminga were assumed for the combined fit, including Monogem. The spectra for both sources assuming with the halo model are shown in Figure 3. The spectral shape for $\mathrm{TeV}$ energy ranges shows a preference in curvature for both Geminga and Monogem.

\section{Acknowledgments}

We acknowledge the support from: the US National Science Foundation (NSF); the US Department of Energy Office of High-Energy Physics; the Laboratory Directed Research and Development (LDRD) program of Los Alamos National Laboratory; Consejo Nacional de Ciencia y Tecnología (CONACyT), México, grants 271051, 232656, 260378, 179588, 254964, 258865, 243290, 132197. A1-S-46288, A1-S-22784, cátedras 873, 1563, 341, 323, Red HAWC, México; DGAPA-UNAM grants IG101320, IN111716-3, IN111419, IA102019, IN110621, IN110521; VIEP-BUAP; PIFI 2012, 2013, PROFOCIE 2014, 2015; the University of Wisconsin Alumni Research Foundation; the Institute of Geophysics, Planetary Physics, and Signatures at Los Alamos National Laboratory; Polish Science Centre grant, DEC-2017/27/B/ST9/02272; Coordinación de la Investigación Científica de la Universidad Michoacana; Royal Society - Newton Advanced Fellowship 180385; Generalitat Valenciana, grant CIDEGENT/2018/034; Chulalongkorn University's CUniverse (CUAASC) grant; Coordinación General Académica e Innovación (CGAI-UdeG), PRODEP-SEP UDG-CA499; Institute of Cosmic Ray Research (ICRR), University of Tokyo. H.F. acknowledges support by NASA under award number 80GSFC21M0002. We also acknowledge the significant contributions over many years of Stefan Westerhoff, Gaurang Yodh and Arnulfo Zepeda Dominguez, all deceased members of the HAWC collaboration. Thanks to Scott Delay, Luciano Díaz and Eduardo Murrieta for technical support.

Additionally, R. Torres-Escobedo would like to thank Dr. Nural Akchurin from the Department of Physics \& Astronomy, Texas Tech University, for the continuing support and guidance. E. de la Fuente thanks PRO-SNI 2021 grant. R. Torres thanks UDG-CA-499 for support through the HAWC Collaboration members.

\section{References}

[1] O. Adriani, G.C. Barbarino, G.A. Bazilevskaya, R. Bellotti, M. Boezio, E.A. Bogomolov et al., An anomalous positron abundance in cosmic rays with energies $1.5-100 \mathrm{GeV}$, Nature 458 (2009) . 
[2] M. Ackermann, M. Ajello, A. Allafort, W.B. Atwood, L. Baldini, G. Barbiellini et al., Measurement of Separate Cosmic-Ray Electron and Positron Spectra with the Fermi Large Area Telescope, Physical Review Letters 108 (2012) .

[3] L. Accardo, M. Aguilar, D. Aisa, B. Alpat, A. Alvino, G. Ambrosi et al., High Statistics Measurement of the Positron Fraction in Primary Cosmic Rays of 0.5-500 GeV with the Alpha Magnetic Spectrometer on the International Space Station, Physical Review Letters 113 (2014) .

[4] D. Hooper, P. Blasi and P.D. Serpico, Pulsars as the sources of high energy cosmic ray positrons, Journal of Cosmology and Astroparticle Physics 2009 (2009) .

[5] D. Malyshev, I. Cholis and J.D. Gelfand, FERMI GAMMA-RAY HAZE VIA DARK MATTER AND MILLISECOND PULSARS, The Astrophysical Journal 722 (2010) .

[6] A.U. Abeysekara, A. Albert, R. Alfaro, C. Alvarez, J.D. Álvarez, R. Arceo et al., The 2HWC HAWC Observatory Gamma-Ray Catalog, The Astrophysical Journal 843 (2017) .

[7] A.U. Abeysekara, A. Albert, R. Alfaro, C. Alvarez, J.D. Álvarez, R. Arceo et al., Extended gamma-ray sources around pulsars constrain the origin of the positron flux at Earth, Science 358 (2017).

[8] G. Giacinti, A.M. Mitchell, R. López-Coto, V. Joshi, R.D. Parsons and J.A. Hinton, Halo fraction in TeV-bright pulsar wind nebulae, Astronomy and Astrophysics 636 (2020) .

[9] M. Di Mauro, S. Manconi and F. Donato, Detection of a $\gamma$-ray halo around Geminga with the Fermi-LAT data and implications for the positron flux, Physical Review D 100 (2019) .

[10] F. Aharonian, Q. An, Axikegu, L. Bai, Y. Bai, Y. Bao et al., Extended Very-High-Energy Gamma-Ray Emission Surrounding PSR J $0622+3749$ Observed by LHAASO-KM2A, Physical Review Letters 126 (2021) .

[11] A.U. Abeysekara, A. Albert, R. Alfaro, C. Alvarez, J.D. Álvarez, J.R.A. Camacho et al., Measurement of the Crab Nebula Spectrum Past $100 \mathrm{TeV}$ with HAWC, The Astrophysical Journal 881 (2019) . 


\section{Full Authors List: HAWC Collaboration}

A.U. Abeysekara ${ }^{48}$, A. Albert ${ }^{21}$, R. Alfaro ${ }^{14}$, C. Alvarez $^{41}$, J.D. Álvarez ${ }^{40}$, J.R. Angeles Camacho ${ }^{14}$, J.C. Arteaga-Velázquez ${ }^{40}$, K. P. Arunbabu $^{17}$, D. Avila Rojas ${ }^{14}$, H.A. Ayala Solares ${ }^{28}$, R. Babu ${ }^{25}$, V. Baghmanyan ${ }^{15}$, A.S. Barber ${ }^{48}$, J. Becerra Gonzalez ${ }^{11}$, E. BelmontMoreno $^{14}$, S.Y. BenZvi ${ }^{29}$, D. Berley ${ }^{39}$, C. Brisbois ${ }^{39}$, K.S. Caballero-Mora ${ }^{41}$, T. Capistrán ${ }^{12}$, A. Carramiñana ${ }^{18}$, S. Casanova ${ }^{15}$, O. Chaparro-Amaro $^{3}$, U. Cotti ${ }^{40}$, J. Cotzomi $^{8}$, S. Coutiño de León ${ }^{18}$, E. De la Fuente ${ }^{46}$, C. de León ${ }^{40}$, L. Diaz-Cruz ${ }^{8}$, R. Diaz Hernandez ${ }^{18}$, J.C. Díaz-Vélez ${ }^{46}$, B.L. Dingus ${ }^{21}$, M. Durocher ${ }^{21}$, M.A. DuVernois ${ }^{45}$, R.W. Ellsworth ${ }^{39}$, K. Engel ${ }^{39}$, C. Espinoza ${ }^{14}$, K.L. Fan ${ }^{39}$, K. Fang $^{45}$, M. Fernández Alonso ${ }^{28}$, B. Fick ${ }^{25}$, H. Fleischhack ${ }^{51,11,52}$, J.L. Flores ${ }^{46}$, N.I. Fraija ${ }^{12}$, D. Garcia ${ }^{4}$, J.A. García-González ${ }^{20}$, J. L. García-Luna ${ }^{46}$, G. García-Torales ${ }^{46}$, F. Garfias ${ }^{12}$, G. Giacinti ${ }^{22}$, H. Goksu ${ }^{22}$, M.M. González ${ }^{12}$, J.A. Goodman ${ }^{39}$, J.P. Harding ${ }^{21}$, S. Hernandez ${ }^{14}$, I. Herzog ${ }^{25}$, J. Hinton ${ }^{22}$, B. Hona ${ }^{48}$, D. Huang ${ }^{25}$, F. Hueyotl-Zahuantitla ${ }^{41}$, C.M. Hui ${ }^{23}$, B. Humensky ${ }^{39}$, P. Hüntemeyer ${ }^{25}$, A. Iriarte ${ }^{12}$, A. Jardin-Blicq ${ }^{22,49,50}$, H. Jhee ${ }^{43}$, V. Joshi ${ }^{7}$, D. Kieda ${ }^{48}$, G J. Kunde ${ }^{21}$, S. Kunwar ${ }^{22}$, A. Lara ${ }^{17}$, J. Lee ${ }^{43}$, W.H. Lee ${ }^{12}$, D. Lennarz ${ }^{9}$, H. León Vargas ${ }^{14}$, J. Linnemann ${ }^{24}$, A.L. Longinotti ${ }^{12}$, R. López-Coto ${ }^{19}$, G. Luis-Raya ${ }^{44}$, J. Lundeen ${ }^{24}$, K. Malone ${ }^{21}$, V. Marandon $^{22}$, O. Martinez ${ }^{8}$, I. Martinez-Castellanos ${ }^{39}$, H. Martínez-Huerta ${ }^{38}$, J. Martínez-Castro ${ }^{3}$, J.A.J. Matthews ${ }^{42}$, J. McEnery ${ }^{11}$, P. Miranda-Romagnoli $^{34}$, J.A. Morales-Soto ${ }^{40}$, E. Moreno ${ }^{8}$, M. Mostafá ${ }^{28}$, A. Nayerhoda ${ }^{15}$, L. Nellen ${ }^{13}$, M. Newbold ${ }^{48}$, M.U. Nisa ${ }^{24}$, R. Noriega-Papaqui ${ }^{34}$, L. Olivera-Nieto ${ }^{22}$, N. Omodei ${ }^{32}$, A. Peisker ${ }^{24}$, Y. Pérez Araujo ${ }^{12}$, E.G. Pérez-Pérez ${ }^{44}$, C.D. Rho ${ }^{43}$, C. Rivière ${ }^{39}$, D. Rosa-Gonzalez ${ }^{18}$, E. Ruiz-Velasco ${ }^{22}$, J. Ryan ${ }^{26}$, H. Salazar ${ }^{8}$, F. Salesa Greus ${ }^{15,53}$, A. Sandoval ${ }^{14}$, M. Schneider ${ }^{39}$, H. Schoorlemmer ${ }^{22}$, J. Serna-Franco ${ }^{14}$, G. Sinnis ${ }^{21}$, A.J. Smith ${ }^{39}$, R.W. Springer ${ }^{48}$, P. Surajbali ${ }^{22}$, I. Taboada ${ }^{9}$, M. Tanner ${ }^{28}$, K. Tollefson ${ }^{24}$, I. Torres ${ }^{18}$, R. Torres-Escobedo $^{30}$, R. Turner ${ }^{25}$, F. Ureña-Mena ${ }^{18}$, L. Villaseñor ${ }^{8}$, X. Wang ${ }^{25}$, I.J. Watson ${ }^{43}$, T. Weisgarber ${ }^{45}$, F. Werner ${ }^{22}$, E. Willox ${ }^{39}$, J. Wood ${ }^{23}$, G.B. Yodh ${ }^{35}$, A. Zepeda ${ }^{4}$, H. Zhou ${ }^{30}$

${ }^{1}$ Barnard College, New York, NY, USA, ${ }^{2}$ Department of Chemistry and Physics, California University of Pennsylvania, California, PA, USA, ${ }^{3}$ Centro de Investigación en Computación, Instituto Politécnico Nacional, Ciudad de México, México, ${ }^{4}$ Physics Department, Centro de Investigación y de Estudios Avanzados del IPN, Ciudad de México, México, ${ }^{5}$ Colorado State University, Physics Dept., Fort Collins, CO, USA, ${ }^{6}$ DCI-UDG, Leon, Gto, México, ${ }^{7}$ Erlangen Centre for Astroparticle Physics, Friedrich Alexander Universität, Erlangen, BY, Germany, ${ }^{8}$ Facultad de Ciencias Físico Matemáticas, Benemérita Universidad Autónoma de Puebla, Puebla, México, ${ }^{9}$ School of Physics and Center for Relativistic Astrophysics, Georgia Institute of Technology, Atlanta, GA, USA, ${ }^{10}$ School of Physics Astronomy and Computational Sciences, George Mason University, Fairfax, VA, USA, ${ }^{11}$ NASA Goddard Space Flight Center, Greenbelt, MD, USA, ${ }^{12}$ Instituto de Astronomía, Universidad Nacional Autónoma de México, Ciudad de México, México, ${ }^{13}$ Instituto de Ciencias Nucleares, Universidad Nacional Autónoma de México, Ciudad de México, México, ${ }^{14}$ Instituto de Física, Universidad Nacional Autónoma de México, Ciudad de México, México, ${ }^{15}$ Institute of Nuclear Physics, Polish Academy of Sciences, Krakow, Poland, ${ }^{16}$ Instituto de Física de São Carlos, Universidade de São Paulo, São Carlos, SP, Brasil, ${ }^{17}$ Instituto de Geofísica, Universidad Nacional Autónoma de México, Ciudad de México, México, ${ }^{18}$ Instituto Nacional de Astrofísica, Óptica y Electrónica, Tonantzintla, Puebla, México, ${ }^{19}$ INFN Padova, Padova, Italy, ${ }^{20}$ Tecnologico de Monterrey, Escuela de Ingeniería y Ciencias, Ave. Eugenio Garza Sada 2501, Monterrey, N.L., 64849, México, ${ }^{21}$ Physics Division, Los Alamos National Laboratory, Los Alamos, NM, USA, ${ }^{22}$ Max-Planck Institute for Nuclear Physics, Heidelberg, Germany, ${ }^{23}$ NASA Marshall Space Flight Center, Astrophysics Office, Huntsville, AL, USA, ${ }^{24}$ Department of Physics and Astronomy, Michigan State University, East Lansing, MI, USA, ${ }^{25}$ Department of Physics, Michigan Technological University, Houghton, MI, USA, ${ }^{26}$ Space Science Center, University of New Hampshire, Durham, NH, USA, ${ }^{27}$ The Ohio State University at Lima, Lima, OH, USA, ${ }^{28}$ Department of Physics, Pennsylvania State University, University Park, PA, USA, ${ }^{29}$ Department of Physics and Astronomy, University of Rochester, Rochester, NY, USA, ${ }^{30}$ Tsung-Dao Lee Institute and School of Physics and Astronomy, Shanghai Jiao Tong University, Shanghai, China, ${ }^{31}$ Sungkyunkwan University, Gyeonggi, Rep. of Korea, ${ }^{32}$ Stanford University, Stanford, CA, USA, ${ }^{33}$ Department of Physics and Astronomy, University of Alabama, Tuscaloosa, AL, USA, ${ }^{34}$ Universidad Autónoma del Estado de Hidalgo, Pachuca, Hgo., México, ${ }^{35}$ Department of Physics and Astronomy, University of California, Irvine, Irvine, CA, USA, ${ }^{36}$ Santa Cruz Institute for Particle Physics, University of California, Santa Cruz, Santa Cruz, CA, USA, ${ }^{37}$ Universidad de Costa Rica, San José, Costa Rica, ${ }^{38}$ Department of Physics and Mathematics, Universidad de Monterrey, San Pedro Garza García, N.L., México, ${ }^{39}$ Department of Physics, University of Maryland, College Park, MD, USA, ${ }^{40}$ Instituto de Física y Matemáticas, Universidad Michoacana de San Nicolás de Hidalgo, Morelia, Michoacán, México, ${ }^{41}$ FCFM-MCTP, Universidad Autónoma de Chiapas, Tuxtla Gutiérrez, Chiapas, México, ${ }^{42}$ Department of Physics and Astronomy, University of New Mexico, Albuquerque, NM, USA, ${ }^{43}$ University of Seoul, Seoul, Rep. of Korea, ${ }^{44}$ Universidad Politécnica de Pachuca, Pachuca, Hgo, México, ${ }^{45}$ Department of Physics, University of Wisconsin-Madison, Madison, WI, USA, ${ }^{46}$ CUCEI, CUCEA, Universidad de Guadalajara, Guadalajara, Jalisco, México, ${ }^{47}$ Universität Würzburg, Institute for Theoretical Physics and Astrophysics, Würzburg, Germany, ${ }^{48}$ Department of Physics and Astronomy, University of Utah, Salt Lake City, UT, USA, ${ }^{49}$ Department of Physics, Faculty of Science, Chulalongkorn University, Pathumwan, Bangkok 10330, Thailand, ${ }^{50}$ National Astronomical Research Institute of Thailand (Public Organization), Don Kaeo, MaeRim, Chiang Mai 50180, Thailand, ${ }^{51}$ Department of Physics, Catholic University of America, Washington, DC, USA, ${ }^{52}$ Center for Research and Exploration in Space Science and Technology, NASA/GSFC, Greenbelt, MD, USA, ${ }^{53}$ Instituto de Física Corpuscular, CSIC, Universitat de València, Paterna, Valencia, Spain 